\title{
Long-term Effects of Androgen Deprivation in a Patient with Spinal and Bulbar Muscular Atrophy - A Case Report with 14 Years of Follow-up
}

\author{
Yasuhiro Hijikata ${ }^{1}$, Atsushi Hashizume ${ }^{1}$, Shinichiro Yamada ${ }^{1}$, Daisuke Ito $^{1}$, Haruhiko Banno ${ }^{1}$, \\ Keisuke Suzuki $^{1,2}$, Gen Sobue $^{3}$ and Masahisa Katsuno ${ }^{1}$
}

\begin{abstract}
:
Spinal and bulbar muscular atrophy (SBMA) is a progressive hereditary neuromuscular disease caused by the testosterone-dependent accumulation of pathogenic polyglutamine-expanded androgen receptor protein. A 41-year-old man with SBMA received the androgen deprivation agent leuprorelin acetate for 7 years in clinical trials and underwent castration following the trial. Suppression of testosterone levels for 14 years resulted in a slower disease progression, as measured prospectively with quantitative measurements, than the historical control data reported in previous studies. This suggests that long-term androgen deprivation delays disease progression in SBMA.
\end{abstract}

Key words: spinal and bulbar muscular atrophy, androgen deprivation, leuprorelin acetate, disease modifying therapies

(Intern Med 58: 2231-2234, 2019)

(DOI: 10.2169/internalmedicine.1592-18)

\section{Introduction}

Spinal and bulbar muscular atrophy (SBMA), or Kennedy's disease, is an X-linked, adult-onset, slowly progressive neuromuscular disease characterized by bulbar and limb muscle weakness (1-3). SBMA is caused by the expansion of a CAG triplet repeat, encoding a polyglutamine tract, in the first exon of the androgen receptor gene (4). The testosterone-dependent nuclear accumulation of the polyglutamine-expanded androgen receptor protein is central to its pathogenesis (5). Although the disease-modifying effect of androgen deprivation therapy has been demonstrated in clinical trials, its long-term effect has not yet been confirmed (6-8).

We herein report the clinical course of a patient with SBMA whose serum testosterone levels had been suppressed below the lower normal limit for 14 years.

\section{Case Report}

A 41-year-old Japanese man gradually realized that he had mild muscle weakness in both lower limbs. He visited our hospital six months after the onset of subjective weakness. He also had a hand tremor that had developed five years before the onset of muscle weakness. On a neurological examination, the manual muscle strength test results were normal except for mild weakness (Muscle Research Council Scale, 4/5) in the cervical flexor muscle and iliopsoas muscle. Hand tremor and contraction fasciculations were also observed. No abnormal findings were apparent in the sensory system. Tendon reflexes were absent in both lower limbs, and no pathological reflex was present. A genetic test for SBMA revealed 46 CAG repeats (normal: 934 ), and the patient was diagnosed with SBMA.

After being apprised of the potential efficacy and adverse effects of androgen deprivation therapy, the patient agreed to

\footnotetext{
${ }^{1}$ Department of Neurology, Nagoya University Graduate School of Medicine, Japan, ${ }^{2}$ Innovation Center for Clinical Research, National Center for Geriatrics and Gerontology, Japan and ${ }^{3}$ Research Division of Dementia and Neurodegenerative Disease, Nagoya University Graduate School of Medicine, Japan

Received: May 30, 2018; Accepted: February 7, 2019; Advance Publication by J-STAGE: April 17, 2019

Correspondence to Dr. Masahisa Katsuno, ka2no@med.nagoya-u.ac.jp
} 
A

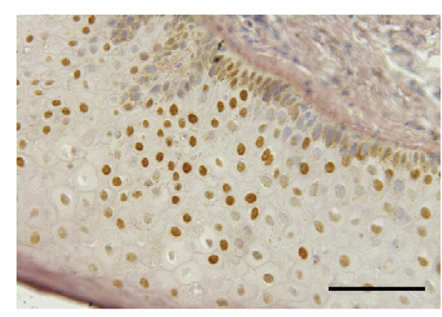

D

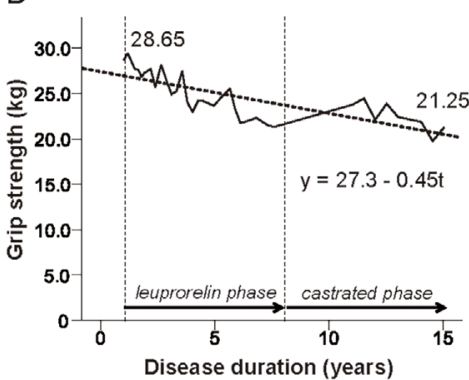

G

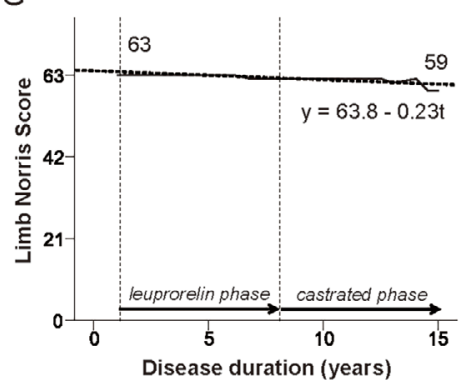

B

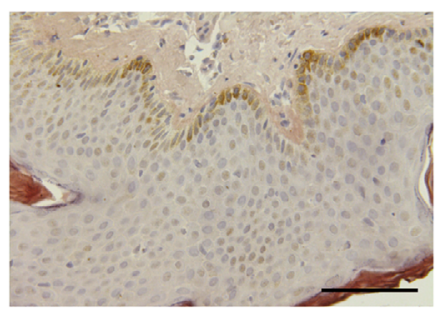

E

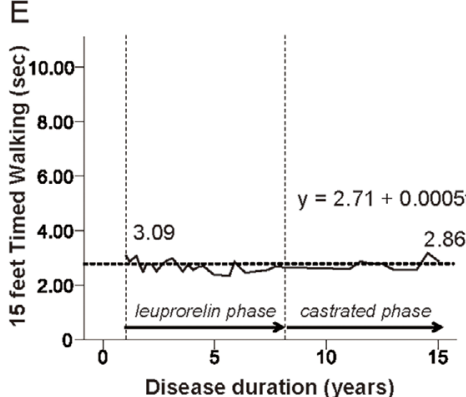

$\mathrm{H}$

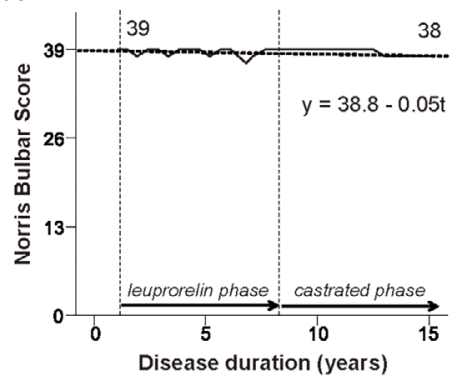

C

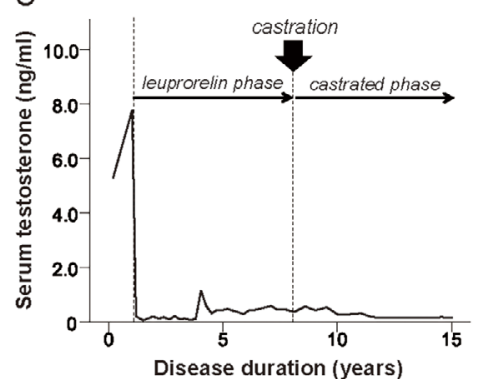

$\mathrm{F}$

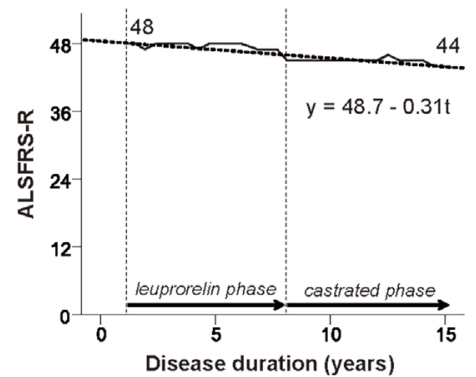

Figure. Changes in the nuclear accumulation of mutant androgen receptor in scrotal skin and longitudinal changes in serum testosterone levels, motor function indices, and their approximation straight line using the least squares method in consideration of disease duration. (A, B) The mutant androgen receptor accumulation in the scrotal skin of the patient before and 12 weeks after leuprorelin treatment. Bar $=25 \mu \mathrm{m}$. (C) The serum testosterone level, (D) Grip strength, (E) 15-foot timed walking, (F) ALSFRS-R, (G) Limb Norris score, (H) Norris bulbar score. ALSFRS-R: revised Amyotrophic Lateral Sclerosis Functional Rating Scale

participate in a phase 2 clinical trial of androgen deprivation with leuprorelin acetate, an agonist of gonadotropinreleasing hormone, followed by an open-label trial where he received leuprorelin acetate (registered with UMIN 000000474) (6). The frequency of cells that showed immunoreactivity for $1 \mathrm{C} 2$, an anti-polyglutamine antibody, in the scrotal skin was decreased by $78 \%$, from $77.3 \%$ to $17.0 \%$ following 48-week leuprorelin treatment (Figure A and B) (9). The patient also participated in a subsequent open-label extension trial in our hospital. Altogether, the patient continued receiving leuprorelin acetate for 7 years with his written informed consent. Upon completion of the extension trial, we explained to the patient that no medication other than rehabilitation and symptomatic treatment had been approved for patients with SBMA. However, he underwent castration at another hospital under private practice, at his own will and cost, without any prior mention to us. His serum testosterone level had been suppressed con- tinuously for approximately 14 years without periods of recovery (10). During this period, a prospective evaluation was made of his motor function indices, including hand grip strength, 15-foot timed walking test result, the revised Amyotrophic Lateral Sclerosis Functional Rating Scale (ALSFRS-R), Limb Norris Score (LNS), and Norris Bulbar Score (NBS), as well as biochemical parameters, as a part of our observational research. This research had been approved by the ethics committee of Nagoya University, and we obtained written informed consent from the patient as well.

The serum testosterone levels had been kept below the reference value since the patient started receiving leuprorelin treatment (Figure C). The rate of decrease in grip strength per year was $0.45 \mathrm{~kg}$, which was $73 \%$ lower than the result shown by a longitudinal study of the natural history of patients with SBMA (Figure D, Table) (11). The 15-foot timed walking test demonstrated that the patient's gait ability had been maintained throughout the observation period (Fig- 
Table. Comparisons between Annual Changes in the Present Case and Those Reported in Natural History Studies.

\begin{tabular}{lccc}
\hline & \multicolumn{3}{c}{ Annual changes of outcome measures } \\
\cline { 2 - 4 } & $\begin{array}{l}\text { Historical } \\
\text { control \#1 }\end{array}$ & $\begin{array}{c}\text { Historical } \\
\text { control \#2 }\end{array}$ & Present case \\
& $-1.7 \pm 3.0$ & N/D & -0.45 \\
\hline Grip strength (kg) & $+1.00 \pm 3.37$ & N/D & +0.00 \\
Timed walking (s) & $-1.1 \pm 0.9$ & $-0.7 \pm 0.3$ & -0.3 \\
ALSFRS-R & $-2.2 \pm 1.6$ & $-0.5 \pm 0.3$ & -0.2 \\
Limb Norris Score & $-1.0 \pm 0.9$ & $-0.7 \pm 0.4$ & -0.0 \\
Norris Bulbar Sore & &
\end{tabular}

Historical control \#1, Reference 11 and Historical control \#2, Reference 12 are described in the table. ALSFRS-R: revised Amyotrophic Lateral Sclerosis Functional Rating Scale

ure E). The patient also maintained high functional scores in the ALSFRS-R, LNS, and NBS (Figure F-H). The changes in biochemical parameters were as follows: serum creatinine, 0.5 to $0.33 \mathrm{mg} / \mathrm{dL}$ (normal $0.6-1.1 \mathrm{mg} / \mathrm{dL}$ ); creatine kinase, 394 to $483 \mathrm{U} / \mathrm{L}$ (normal 45-245 U/L); aspartate aminotransferase, 55 to $49 \mathrm{U} / \mathrm{L}$ (normal $<41 \mathrm{U} / \mathrm{L}$ ); alanine aminotransferase, 109 to $76 \mathrm{U} / \mathrm{L}$ (normal $<45 \mathrm{U} / \mathrm{L}$ ); total cholesterol, 247 to $275 \mathrm{mg} / \mathrm{dL}$ (normal 120-220 mg/dL); and hemoglobin A1c, $7.3 \%$ to $5.7 \%$ (normal 4.9-6.0\%).

During the observation period, the patient complained of decreased libido. However, serious events, such as falls, bone fractures, and pneumonia, did not occur.

\section{Discussion}

To our knowledge, this is the longest follow-up to date of a patient with SBMA whose serum testosterone level had been suppressed below the lower normal limit. The progression rate of motor dysfunction evaluated with quantitative outcome measurements was slower than that of controls reported in previous studies (Table) $(11,12)$, suggesting longterm disease modification by androgen deprivation.

Several side effects of androgen deprivation therapy, such as hot flashes and sexual dysfunction (13), increased risks of fracture (14), metabolic syndrome (15), and thromboembolic events $(16,17)$, have been reported in patients with prostate cancer. However, for patients with SBMA, it is difficult to clearly distinguish the symptoms associated with SBMA from the adverse effects associated with androgen deprivation therapy because patients with SBMA often have partial androgen insensitivity due to androgen receptor gene mutations (5). In the present case, the patient had mild sexual dysfunction but did not develop serious diseases, such as myocardial infarction, during the observation period.

Clinical trials of disease-modifying therapies for neurodegenerative diseases frequently exhibit poor efficacy despite early success in animal models (5). Early intervention before irreversible changes occur in the structure or function is thought to be key to the success of disease-modifying treatments in neurodegenerative diseases (18). In the present case, the initiation of androgen deprivation therapy from the very early stage of the disease (six months from the onset of muscle weakness) might have been one reason for the slow progression of the disease.

In cases of slowly progressive neurodegenerative diseases, such as SBMA, the long-term observation, which is difficult to accomplish in clinical trials, is required in order to detect the efficacy of disease-modifying therapies. In the present case, we conducted a prospective evaluation of the motor function indices of a patient whose serum testosterone level had been suppressed below the lower normal limit for 14 years. Although such long-term observation will likely aid in evaluating the efficacy of surgical castration in patients with SBMA, we believe that similar cases will not occur in Japan because leuprorelin acetate, which shows an androgen deprivation effect equal to that of surgical castration, has already been approved as a new treatment for patients with SBMA by the Pharmaceuticals and Medical Devices Agency (7). We do not recommend castration be performed in patients with SBMA because castration has not been confirmed to have medical efficacy and safety. Further studies will be required to confirm the long-term and real-world efficacy of leuprorelin acetate in the early stages of SBMA.

\section{Author's disclosure of potential Conflicts of Interest (COI).}

Gen Sobue: Research funding, Takeda Pharmaceutical; Patent royalties/licensing fees, Takeda Pharmaceutical. Masahisa Katsuno: Fees for promotional materials, Takeda Pharmaceutical; Research funding, Takeda Pharmaceutical; Patent royalties/licensing fees, Takeda Pharmaceutical.

\section{Financial Supports}

This work was funded by a Grant-in-Aid (KAKENHI) from the Ministry of Education, Culture, Sports, Science and Technology of Japan (No. 17H04195); grants from the Japan Agency for Medical Research and Development (No. 18ek0109359h0001 and 17ek0109221h0001); and a grant from the Naito Foundation.

Dr. Hijikata is supported by a grant from the Kanae Science Foundation for the Promotion of Medical Science.

Dr. Hashizume is supported by KAKENHI grants from MEXT/JSPS, Japan (No. 15K09311).

Dr. Sobue serves as a scientific advisory board member for the Kanae Science Foundation for the Promotion of Medical Science, Naito Science Foundation; an advisory board member of Brain; and an editorial board member of Degenerative Neurological and Neuromuscular disease, the Journal of Neurology, and Amyotrophic Lateral Sclerosis and Frontotemporal Degeneration. $\mathrm{He}$ is supported by the "Integrated Research on Neuropsychiatric Disorders" and "Integrated Research on Depression, Dementia and Development Disorders" projects carried out under the Strategic Research Program for Brain Sciences and Brain/MINDS of MEXT, Japan; grants from the Japan Agency for Medical Research and Development; a MEXT Grant-in-aid project, Scientific Research on Innovation Area (Brain Protein Aging and Dementia control).

Dr. Katsuno is supported by KAKENHI grants from MEXT/ JSPS, Japan (Nos. 17H04195 and 16K15480); grants from Japan Agency for Medical Research and Development (Nos. 18ek 
0109359h0001, 17ek0109221h0001, 16dk0207026h0001, and 15 ek0109165); a grant from the Naito Foundation; a grant from the Uehara Memorial Foundation; and a grant from the Hori Sciences and Arts Foundation.

\section{References}

1. Kennedy WR, Alter M, Sung JH. Progressive proximal spinal and bulbar muscular atrophy of late onset. A sex-linked recessive trait. Neurology 18: 671-680, 1968.

2. Sobue G, Hashizume Y, Mukai E, Hirayama M, Mitsuma T, Takahashi A. X-linked recessive bulbospinal neuronopathy. A clinicopathological study. Brain 112: 209-232, 1989.

3. Fischbeck KH. Kennedy disease. J Inherit Metab Dis 20: 152-158, 1997.

4. La Spada AR, Wilson EM, Lubahn DB, Harding AE, Fischbeck $\mathrm{KH}$. Androgen receptor gene mutations in X-linked spinal and bulbar muscular atrophy. Nature 352: 77-79, 1991.

5. Katsuno M, Tanaka F, Adachi H, et al. Pathogenesis and therapy of spinal and bulbar muscular atrophy (SBMA). Prog Neurobiol 99: 246-256, 2012.

6. Banno H, Katsuno M, Suzuki K, et al. Phase 2 trial of leuprorelin in patients with spinal and bulbar muscular atrophy. Ann Neurol 65: 140-150, 2009.

7. Katsuno M, Banno H, Suzuki K, et al.; Japan SBMA Interventional Trial for TAP-144-SR (JASMITT) study group. Efficacy and safety of leuprorelin in patients with spinal and bulbar muscular atrophy (JASMITT study): a multicentre, randomised, doubleblind, placebo-controlled trial. Lancet Neurol 9: 875-884, 2010.

8. Hashizume A, Katsuno M, Suzuki K, et al. Long-term treatment with leuprorelin for spinal and bulbar muscular atrophy: natural history-controlled study. J Neurol Neurosurg Psychiatry 88: 10261032, 2017.

9. Trottier Y, Lutz Y, Stevanin G, et al. Polyglutamine expansion as a pathological epitope in Huntington's disease and four dominant cerebellar ataxias. Nature 378: 403-406, 1995.

10. van der Sluis TM, Bui HN, Meuleman EJ, et al. Lower testosterone levels with luteinizing hormone-releasing hormone agonist therapy than with surgical castration: new insights attained by mass spectrometry. J Urol 187: 1601-1606, 2012.

11. Hashizume A, Katsuno M, Banno H, et al. Longitudinal changes of outcome measures in spinal and bulbar muscular atrophy. Brain 135: 2838-2848, 2012.

12. Hashizume A, Katsuno M, Suzuki K, et al. A functional scale for spinal and bulbar muscular atrophy: cross-sectional and longitudinal study. Neuromuscul Disord 25: 554-562, 2015.

13. Sethi R, Sanfilippo N. Six-month depot formulation of leuprorelin acetate in the treatment of prostate cancer. Clin Interv Aging 4: 259-267, 2009.

14. Shahinian VB, Kuo YF, Freeman JL, Goodwin JS. Risk of fracture after androgen deprivation for prostate cancer. N Engl J Med 352: 154-164, 2005.

15. Saylor PJ, Smith MR. Metabolic complications of androgen deprivation therapy for prostate cancer. J Urol 181: 1998-2006, 2009.

16. Van Hemelrijck M, Garmo H, Holmberg L, et al. Absolute and relative risk of cardiovascular disease in men with prostate cancer: results from the Population-Based PCBaSe Sweden. J Clin Oncol 28: 3448-3456, 2010.

17. Hershman DL, Unger JM, Wright JD, et al. Adverse health events following intermittent and continuous androgen deprivation in patients with metastatic prostate cancer. JAMA Oncol 2: 453-461, 2016.

18. Andrieu S, Coley N, Lovestone S, Aisen PS, Vellas B. Prevention of sporadic Alzheimer's disease: lessons learned from clinical trials and future directions. Lancet Neurol 14: 926-944, 2015.

The Internal Medicine is an Open Access journal distributed under the Creative Commons Attribution-NonCommercial-NoDerivatives 4.0 International License. To view the details of this license, please visit (https://creativecommons.org/licenses/ by-nc-nd/4.0/).

(C) 2019 The Japanese Society of Internal Medicine Intern Med 58: 2231-2234, 2019 